\title{
DIENTES DE UN ENIGMÁTICO DINOSAURIO ORNITÓPODO EN EL CRETÁCICO INFERIOR DE BURGOS (ESPAÑA)
}

\author{
Fidel TORCIDA FERNÁNDEZ-BALDOR ${ }^{1,2}$, José \\ Ignacio RUIZ-OMEÑACA ${ }^{3}$, Luis Ángel IZQUIER- \\ DO MONTERO ${ }^{1,2}$, Diego MONTERO HUERTA ${ }^{1,2}$, \\ Gustavo PÉREZ MARTÍNEZ ${ }^{1,2}$, Pedro HUERTA \\ HURTADO ${ }^{1,2}$ y Víctor URIÉN MONTERO ${ }^{1,2}$
}

\author{
${ }^{1}$ Colectivo Arqueológico-Paleontológico de Salas (C.A.S.). Plaza Jesús Apari- \\ cio, 9. 09600 Salas de los Infantes, Burgos. www.colectivosalas.com \\ ${ }^{2}$ Museo de Dinosaurios. Plaza Jesús Aparicio, 9. 09600 Salas de los Infantes, \\ Burgos. www.salasdelosinfantes.net/museo \\ 3 Área y Museo de Paleontología, Facultad de Ciencias, Universidad de Zara- \\ goza, 50009 Zaragoza.
}

\begin{abstract}
Torcida Fernández-Baldor, F., Ruiz-Omeñaca, J. I., Izquierdo Montero, L. A., Montero Huerta, D., Pérez Martínez, G., Huerta Hurtado, P. \& Urién Montero, V. 2005. Dientes de un enigmático dinosaurio ornitópodo en el Cretácico Inferior de Burgos (España). [Enigmatic ornithopod teeth from the Lower Cretaceous of Burgos (Spain).] Revista Española de Paleontología, N.E. X, 73-81. ISSN 0213-6937.
\end{abstract}

\begin{abstract}
Premaxillary, maxillary and dentary teeth from the Lower Cretaceous (upper Barremian-Aptian, Castrillo de la Reina Formation) El Peñascal site, near to Salas de los Infantes (Burgos, northern Spain), are described. After a comparison with teeth of all ornithopod groups, it is deduced that they belong to an euornithopod, and that they could have belonged to an "hypsilophodontid" or a "rhabdomorphan". The teeth present differences with the described ones in all the genera of these groups, and are provisionally assigned to Euornithopoda indet. This taxon may represent a new undescribed euornithopod in the European Lower Cretaceous.
\end{abstract}

Keywords: teeth, Ornithischia, Euornithopoda, "Rhabdomorpha", "Hypsilophodontidae".

\section{RESUMEN}

Se describen dientes premaxilares, maxilares y mandibulares procedentes del yacimiento de El Peñascal, próximo a Salas de los Infantes (Burgos, Norte de España), y datado en el Cretácico Inferior, Formación Castrillo de la Reina (Barremiense superior-Aptiense). De la comparación con dientes de todos los grupos de ornitópodos, se deduce que son de euornitópodo, y que podrían pertenecer tanto a un "hipsilofodóntido" como a un "rhabdomorfo"; sin embargo, se aprecian diferencias con las características presentes en todos los géneros descritos de estos grupos, por lo que se asignan provisionalmente a Euornithopoda indet. Se propone que este taxón representa un euornitópodo no descrito hasta ahora para el Cretácico Inferior de Europa, y posiblemente constituya un nuevo género y especie.

Palabras clave: dientes, Ornithischia, Euornithopoda, "Rhabdomorpha", "Hypsilophodontidae”.

\section{INTRODUCCIÓN}

El área de Salas de los Infantes (Burgos) es rica en restos fósiles de dinosaurios y otros vertebrados continentales del Cretácico Inferior. Entre la amplia colección de restos de dinosaurios, procedentes de yacimientos de diferentes municipios y depositados en el Museo de Dinosaurios de Salas de los Infantes, destacan por su abundacia y buena conservación los restos de pequeños ornitópodos, de los que hay varios ejemplares de hipsilofodóntidos (Hypsilophodontidae indet., Hypsilophodontidae nov. gen., cf. Hypsilophodon sp.) y driosáuridos (Valdosaurus sp., 
“Camptosaurus" valdensis Lydekker, 1889), la mayoría sin describir (véanse Torcida Fernández-Baldor, 1996; RuizOmeñaca, 2001; Torcida Fernández-Baldor et al., 2003).

El Peñascal está situado en terrenos comunales de Salas de los Infantes, Villanueva de Carazo, Hacinas, La Revilla y Barbadillo del Mercado, a 1,5 km al este de Villanueva de Carazo (Fig. 1). Geológicamente se sitúa en la Cuenca de Cameros Occidental, en arcillas rojas de la Formación Castrillo de la Reina, de edad Barremiense superior-Aptiense (Martín-Closas \& Alonso Millán, 1998). Las arcillas rojas en las que han aparecido los restos se intercalan con cuerpos arenosos, y se interpretan como medios aluviales (Platt, 1986; Mas et al., 1993).

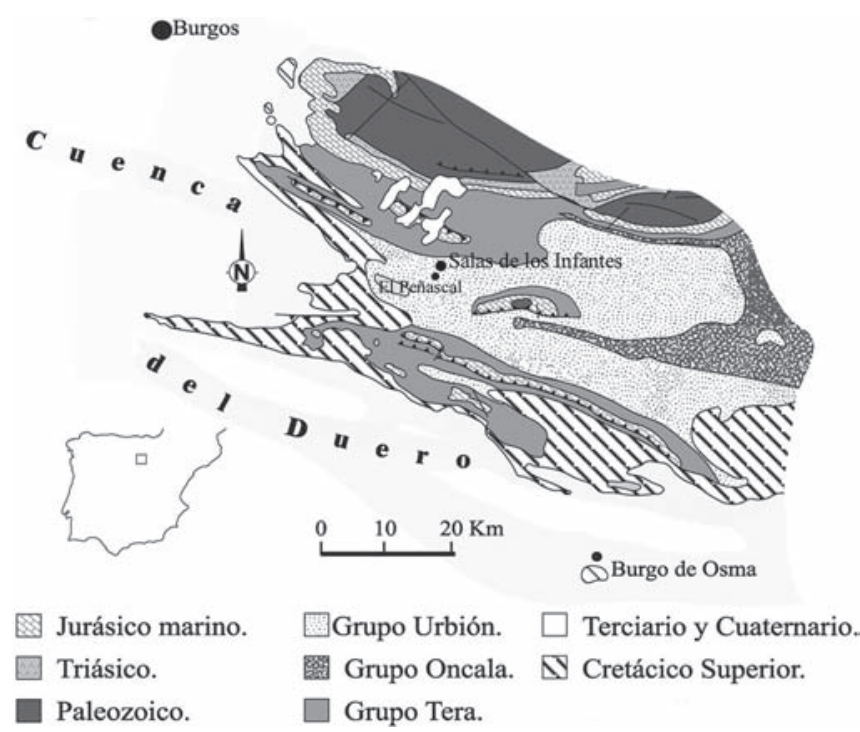

Figura 1. Mapa geológico de la zona objeto de estudio, con la localización del yacimiento de El Peñascal. Modificado de Beuther et al. (1966).

Geologic map of the studied area. The El Peñascal site is marked by a point. Modified from Beuther et al. (1966).

El yacimiento ha proporcionado restos de un ornitópodo con una serie de características que nos hacen suponer que se trata de un nuevo taxón para el Cretácico Inferior europeo, y posiblemente permitan proponer una nueva especie. En este trabajo se presenta una descripción preliminar de la dentición de este ornitópodo.

Los materiales estudiados aquí proceden de una prospección superficial, en la que se recuperaron restos craneales y postcraneales, algunos aparentemente en articulación como un dedo del pie completo; otros restos del yacimiento pertenecen a cocodrilos y tortugas. En 2004 se realizó una excavación que ha proporcionado nuevo material sometido ahora a restauración y consolidación; entre las piezas recuperadas aparecen más dientes premaxilares, maxilares y mandibulares, con características similares a los descritos aquí, y de tamaños diversos.
El yacimiento aparece en una sucesión en la que se intercalan areniscas y lutitas. Las areniscas tienen cantos cuarcíticos dispersos de $2 \mathrm{~cm}$ aproximadamente. Se corresponden con depósitos de canales fluviales de pequeñas dimensiones con anchuras inferiores a $4 \mathrm{~m}$ y espesores en torno a $1 \mathrm{~m}$. Las lutitas son de color rojo y se interpretan como depósitos de llanura de inundación.

Los materiales encontrados han aparecido en un área de $1 \mathrm{~m}^{2}$. El pequeño tamaño de los dientes y las vértebras encontradas hizo imposible reconocer una orientación de los mismos pues parte del material se obtuvo una vez lavado el sedimento. La ausencia de cantos cuarcíticos asociados a los dientes sugiere que no existía una corriente tractiva energética que acumulase los restos óseos durante los desbordamientos. La densidad de un diente puede ser similar a la de un canto de cuarcita del mismo tamaño. En condiciones de flujo similares se depositarían juntos. Esto está apoyado por la falta de evidencias de abrasión. Este hecho no descarta una ligera removilización de los materiales fósiles, pudiendo existir uno o más individuos mezclados. De lo anterior deducimos la existencia de restos de varios individuos, al menos dos, del mismo taxón y diferenciados entre sí por su tamaño diferente.

En trabajos futuros se abordará el estudio de restos óseos postcraneales asociados a los dientes, así como del material fósil extraído en la excavación citada; uno de los objetivos que nos planteamos es la posibilidad de crear un nuevo taxón con los datos obtenidos.

\section{SISTEMÁTICA}

Orden ORNITHISCHIA Seeley, 1887

Hipoorden ORNITHOPODA Marsh, 1881

Suborden EUORNITHOPODA Sereno, 1986 sensu

Weishampel, 1990

\section{EUORNITHOPODA indet.}

Figs. 2 y 3 , Tabla 1

Material: Por el momento, se han recuperado en el yacimiento un total de 16 dientes (un diente premaxilar, siete dientes maxilares y ocho dientes mandibulares). Los dientes se conservan en el «Museo de Dinosaurios» de Salas de los Infantes, con las siglas PS-PLS (Paleontología Salas-PeñascaL Salas)

De los 16 dientes, 4 no se han estudiado por su mal estado de conservación o por ser muy fragmentarios. La relación completa de los dientes estudiados es la siguiente:

- Diente premaxilar: PS-PLS,21. (Fig. 2: 2a; Fig. 3: 3a y $3 \mathrm{~b}$; tabla 1)

- Dientes maxilares: PS-PLS,18; PS-PLS,25; PS-PLS,27; PS-PLS,34; PS-PLS,37. (Fig. 2 : 2b, 2c y 2d; Fig. 3: 3c, 3d y 3e; tabla 1)

- Dientes mandibulares: PS-PLS,19; PS-PLS,29; PS-PLS,30; PS-PLS,31; PS-PLS,35; PS-PLS,36. (Fig. 2: 2e y 2f; Fig. 3: 3f, $3 \mathrm{~g}$ y $3 \mathrm{~h}$; tabla 1 ) 


\section{Descripción}

Para la descripción de los dientes se sigue la nomenclatura de Thulborn (1970: 423): «lingual», «mesial», «distal», «adapical» y «oclusal». El término «labial», usado aquí, es equivalente al «bucal» de Thulborn (1970).

Los dientes de El Peñascal que estudiamos aquí corresponden a 5 dientes funcionales, que conservan la raíz y tienen un mayor
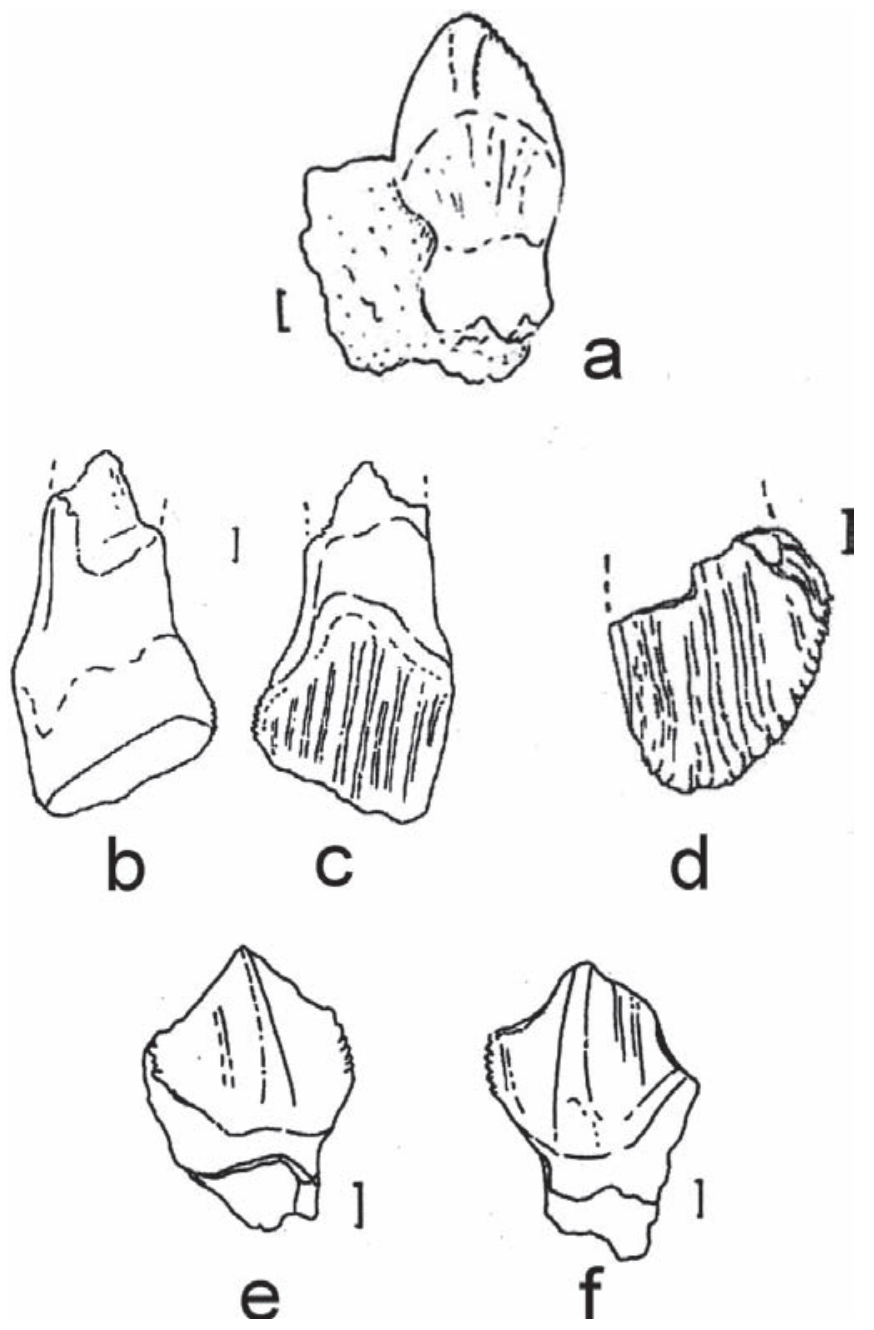

Figura 2. Dientes de El Peñascal (Burgos) Euornithopoda indet. a: Vista labial del diente premaxilar PS-PLS,21. b, c y d, dientes maxilares; b: Vista lingual del diente PS-PLS, 18, con faceta de desgaste. c: Vista labial del diente PS-PLS,18. d: Vista labial del diente PS-PLS,34. e y f, dientes mandibulares en vista lingual. e: Diente PS-PLS-30. f: Diente PS-PLS,35. Medida de referencia: $1 \mathrm{~mm}$.

Teeth from The Peñascal site (Burgos, Spain) Euornithopoda indet. a: Premaxillary tooth PS-PLS,21 in labial view. $\boldsymbol{b}, \boldsymbol{c}$ and $\boldsymbol{d}$, Maxillary teeth. $\boldsymbol{b}$ : Tooth PS-PLS, 18 in lingual view, with wear facet. $\boldsymbol{c}$ : Tooth PS-PLS,18 in labial view. $d$ : Tooth PS-PLS,34 in labial view. $\boldsymbol{e}$ and $f$, Mandibular teeth in lingual view. $\boldsymbol{e}$ : Tooth PS-PLS,30. f: Tooth PS-PLS,35. Scale bar: $1 \mathrm{~mm}$. o menor grado de desgaste oclusal, y a 4 coronas de reemplazamiento, que carecen de desgaste; en esta relación se excluyen 3 dientes que, por rotura o por inclusión en matriz, no puede saberse con exactitud si son funcionales o de reemplazamiento.

Diente premaxilar: El único diente premaxilar, PS-PLS,21, es comprimido labio-lingualmente; no posee ornamentación en el esmalte de la corona (cara labial), y presenta los bordes mesial y distal con dentículos poco desarrollados (Fig. 2a; Fig. 3a y 3b). Los bordes del diente son diferentes entre sí: uno es más ancho que el otro. En vista lateral, la mitad de la corona que corresponde al borde más ancho es convexa, tanto en la cara labial como en la lingual; la mitad de la corona del borde más estrecho es plana en las caras labial y lingual. Como resultado de lo anterior, la corona del diente es asimétrica, lo que se aprecia claramente en vista apical. La raíz es de sección subcircular; en su cara lingual desarrolla un surco ancho en sentido adapical, que se interrumpe en la base de la corona, donde aparecen dos pequeños mamelones laterales.

Dientes maxilares: Los dientes identificados como maxilares (véase discusión), PS-PLS,18; PS-PLS,25; PS-PLS,27; PS-PLS,34; PS-PLS,37, son pequeños, entre 6 y $11 \mathrm{~mm}$ de altura de la corona (tabla 1). De los cinco dientes estudiados hemos podido diferenciar dos dientes funcionales y uno de reemplazamiento. Su corona es ancha y comprimida labio-lingualmente. Aunque tienen esmalte en las caras lingual y labial, está más desarrollado en la cara labial, donde aparecen de 6 a 10 crestas paralelas y subiguales, de las que 4 ó 5 en la parte central de la cara están más marcadas. Los dientes en los que se ha contado menos crestas (tabla 1) presentan el esmalte deteriorado (PS-PLS,27), o el recuento no es exacto y no se puede descartar que haya más crestas, al estar la pieza incluida en matriz (PS-PLS,37). Poseen un borde cóncavo, que se interpreta como mesial, y el otro recto, que se interpreta como distal. Los dos bordes poseen dentículos, en varios de los cuales confluyen las crestas del esmalte. Entre la raíz y la corona se desarrolla un cíngulo bien marcado.

Dientes mandibulares: Los dientes identificados como mandibulares (véase discusión), PS-PLS,19; PS-PLS,29; PS-PLS,30; PS-PLS,31; PS-PLS,35; PS-PLS,36, son también de pequeño tamaño, con valores de altura de la corona que oscilan entre 6,5 y $14,5 \mathrm{~mm}$ (tabla 1 ). De los seis dientes analizados, se ha podido diferenciar 2 funcionales y 3 de reemplazamiento. Presentan esmalte en ambos lados de la corona, aunque en uno de ellos está más desarrollado. En esa cara más esmaltada, que se interpreta como lingual, aparece una cresta primaria muy marcada, en posición central, y una, dos o tres crestas secundarias, mucho menos marcadas, y situadas a los lados de la cresta primaria. Los bordes mesial y distal presentan dentículos. Entre la corona y la raíz hay un cíngulo grueso en la cara lingual, del que parte la cresta central hacia el ápice del diente. La raíz es de sección subcircular y está hueca.

\section{Discusión}

Los dientes de ornitópodo son de formas muy variables, dependiendo del taxón al que pertenezcan y de su posición en las mandíbulas (premaxilar, maxilar y mandibular). 


\begin{tabular}{|c|c|c|c|c|c|}
\hline siglas medidas & $\begin{array}{l}\text { altura } \\
\text { máxima de } \\
\text { la corona }\end{array}$ & $\begin{array}{l}\text { longitud máxima } \\
\text { (antero-posterior) }\end{array}$ & $\begin{array}{c}\text { anchura máxima } \\
\text { (labio-lingual) }\end{array}$ & número de crestas & posición anatómica \\
\hline PS-PLS, 21 & 8.5 & 4.5 & 2 & No presenta & Premaxilar \\
\hline PS-PLS, 18 & 10.5 & 5.5 & 3.5 & 10 & Maxilar izquierdo \\
\hline PS-PLS, 25 & 7.5 & 3.5 & 2 & 8 & Maxilar izquierdo \\
\hline PS-PLS, 27 & 11 & 5.5 & 3.5 & 7 & Maxilar izquierdo \\
\hline PS-PLS,34 & 6 & 5.5 & 3 & 8 & Maxilar \\
\hline PS-PLS,37 & 10 & 5 & - & 6 & Maxilar izquierdo \\
\hline PS-PLS, 19 & 14.5 & 6.5 & 4 & $\begin{array}{c}1 \text { primaria } \\
3 \text { secundarias }\end{array}$ & Mandibular \\
\hline PS-PLS,29 & 8 & 5.5 & 4 & $\begin{array}{c}1 \text { primaria } \\
2 \text { secundarias }\end{array}$ & Mandibular \\
\hline PS-PLS, 30 & 6.5 & 5 & 3 & $\begin{array}{l}1 \text { primaria } \\
1 \text { secundaria }\end{array}$ & Mandibular \\
\hline PS-PLS,31 & 11 & 6 & 4.5 & $\begin{array}{l}1 \text { primaria } \\
1 \text { secundaria }\end{array}$ & Mandibular \\
\hline PS-PLS,35 & 7.5 & 6 & 3.5 & $\begin{array}{c}1 \text { primaria } \\
3 \text { secundarias }\end{array}$ & Mandibular \\
\hline PS-PLS,36 & 10 & 5.5 & 3.5 & $\begin{array}{c}1 \text { primaria } \\
3 \text { secundarias }\end{array}$ & Mandibular \\
\hline
\end{tabular}

Tabla 1. Algunos parámetros estudiados en los dientes del euornitópodo de El Peñascal (Burgos). Medidas en mm. Some parameters studied in the teeth of the euornithopod from the El Peñascal site (Burgos, Spain). Measurements in mm.

Son comprimidos labio-lingualmente y con dentículos en los bordes mesial y distal; el esmalte suele estar más desarrollado en una cara (en los maxilares es la cara labial, y en los mandibulares es la cara lingual), y sobre la cara más esmaltada se desarrollan crestas, mientras que la cara menos esmaltada es convexa y lisa (Weishampel, 1984). Los ornitópodos más basales, como heterodontosáuridos e "hipsilofodóntidos", conservan dientes premaxilares (Sues \& Norman, 1990; Weishampel \& Witmer, 1990). La forma general de los dientes mandibulares y maxilares de El Peñascal permite identificarlos como dientes de ornitópodo.

La ausencia de dientes mudados sugiere que todos los dientes estaban en las mandíbulas/maxilares en el momento de su enterramiento y que podrían proceder de un mismo cráneo. En el yacimiento hay más de un individuo, como muestra el tamaño no coincidente de las piezas óseas y dentales recuperadas; los dientes que se describen en este trabajo por su tamaño, morfología y conservación, pertenecen a un mismo ejemplar.

La presencia de dientes premaxilares indica que el ornitópodo de El Peñascal es basal; los dientes premaxilares desaparecen completamente en Dryomorpha (Dryosaurus + camptosáuridos +"iguanodóntidos" + hadrosaurios; Norman, 2001).

La presencia de dientes premaxilares en "rhabdomorfos" es variable: Zalmoxes robustus (Nopcsa, 1900) y Tenontosaurus tilletti Ostrom, 1970 no tienen pero Tenontosaurus dosii Winkler, Murry \& Jacobs, 1997 sí; en Zalmoxes shquiperorum Weishampel, Jianu, Csiki \& Norman, 2003, Rhabdodon Matheron, 1869, Mochlodon Seeley, 1881 y Muttaburrasaurus Bartholomai \& Molnar, 1981 no puede comprobarse en el material conservado (RuizOmeñaca, 2001; Weishampel et al., 2003). 
Sí que tienen dientes premaxilares los heterodontosáuridos y los "hipsilofodóntidos" (Sues \& Norman, 1990; Weishampel \& Witmer, 1990). Los dientes laterales (maxilares y mandibulares) de los heterodontosáuridos tienen coronas altas con forma de cincel (Weishampel \& Witmer, 1990; Norman \& Barret, 2002); carácter que aparece también en el ceratopsio basal Chaoyangsaurus Zhao, Cheng \& Xu, 1999 y sin ornamentación, por lo que se diferencian de los del ornitópodo de El Peñascal.

Por tanto, el ornitópodo de El Peñascal no es un Dryomorpha ni un Heterodontosauridae, y podría ser un "Hypsilophodontidae" o un "Rhabdomorpha" (en el sentido de Pincemaille, 1997, 1999, y Ruiz-Omeñaca, 2001), ambos euornitópodos. Hay que destacar que ambos grupos son parafiléticos según algunos autores (véase Weishampel et al., 2003; Norman, 2004; Norman et al., 2004), por lo que en este trabajo estos taxones se usan entrecomillados. Aunque Weishampel et al. (2003) aceptan la familia Rhabdodontidae (compuesta por Mochlodon, Rhabdodon, y Zalmoxes Weishampel, Jianu, Csiki \& Norman, 2003), Norman (2004) no la reconoce y lista estos taxones como Iguanodontia basales, por lo que en este trabajo se prefiere utilizar el término "rhabdomorfo" a rhabdodóntido.

Entre los dientes de El Peñascal se han reconocido tres morfologías: A) dientes sin ornamentación, B) dientes con una cresta central muy marcada, y C) dientes con varias crestas secundarias subiguales y paralelas. Los dientes del tipo A pueden identificarse como dientes premaxilares. En los "hipsilofodóntidos" y "rhabdomorfos", sólo los dientes mandibulares presentan, a veces, una cresta primaria desarrollada, por lo que las morfologías B y C se han identificado como dientes mandibulares y dientes maxilares, respectivamente.

Todos los "rhabdomorfos" (Mochlodon, Muttaburrasaurus, Rhabdodon, Tenontosaurus Ostrom, 1970, Zalmoxes) tienen dientes maxilares con numerosas crestas secundarias paralelas y sin cresta primaria, pero los dientes mandibulares tienen una cresta primaria y numerosas crestas secundarias mesiales y distales (Molnar, 1996; Weishampel et al., 2003), diferenciándose del ornitópodo de El Peñascal.

Dentro de "Hypsilophodontidae", familia recientemente puesta en duda al ser parafilética (véase Weishampel et al., 2003), la variabilidad dental es alta. No se conoce la dentición de todos los géneros descritos, así ésta se desconoce en Fulgurotherium Huene, 1932, Loncosaurus Ameghino, 1898 y Notohypsilophodon Martínez, 1998. Sí que se conocen los dientes de Agilisaurus Peng, 1990, Atlascopcosaurus Rich \& Rich, 1989, Bugenasaura Galton, 1995, Drinker Bakker, Galton, Siegwarth \& Filla, 1990, Gasparinisaura Coria \& Salgado, 1996, Gongbusaurus Dong, Zhou \& Zhang, 1983, Hypsilophodon Huxley, 1869, Leaellynasauria Rich \& Rich, 1989, Othnielia Galton, 1977, Orodromeus Horner \& Weishampel, 1988, Parksosaurus
Sternberg, 1937, Phyllodon Thulborn, 1973, Qantassaurus Rich \& Vickers-Rich, 1999, Thescelosaurus Gilmore, 1913, Xiaosaurus Dong \& Tang, 1983, Yandusaurus He, 1979 y Zephyrosaurus Sues, 1980, con los que se van a comparar los dientes de El Peñascal.

Los dientes maxilares de Phyllodon, Orodromeus y Xiaosaurus no tienen ornamentación de crestas longitudinales (Thulborn, 1973; Dong \& Tang, 1983; Galton, 1995), por lo que se diferencian de los dientes de El Peñascal.

Los dientes maxilares de Othnielia y Drinker tienen crestas secundarias (una por cada dentículo) irregulares y con desarrollo variable (Galton, 1983; Bakker et al., 1990); estas crestas están formadas por irregularidades del esmalte (pústulas y crestas sinuosas) alineadas (Bakker et al., 1990), por lo que se diferencian de los de El Peñascal.

Los dientes maxilares de Agilisaurus tienen una cresta primaria redondeada y crestas secundarias muy débiles (Peng, 1992, 1997). Los dientes maxilares de Gasparinisaura tienen una cresta primaria y varias crestas secundarias (Coria \& Salgado, 1996). Los dientes maxilares de Atlascopcosaurus tienen una cresta central, además de, al menos, siete crestas secundarias (Rich \& Rich, 1989; Rich \& Vickers-Rich, 1999). Los dientes maxilares de Bugenasaura y de Thescelosaurus tienen una cresta central marcada y numerosas crestas secundarias (hasta 12 en Bugenasaura, Galton, 1997; hasta 15 en Thescelosaurus, Galton, 1999); además las crestas no son paralelas sino que forman círculos convergentes a ambos lados de la cresta central (Galton, 1999). Todos estos taxones se diferencian de los dientes de El Peñascal por tener una cresta primaria.

Los dientes maxilares de Hypsilophodon tienen normalmente tres crestas secundarias, aunque pueden desarrollarse hasta seis, que pueden o no llegar a la base de la corona (Galton, 1974: 43); en El Peñascal hay mayor número de crestas que llegan a la base de la corona. Los dientes maxilares de Leaellynasauria tienen hasta cinco crestas secundarias (Rich \& Rich, 1989; Rich \& VickersRich, 1999). Estos taxones se diferencian de los dientes de El Peñascal por presentar un numero bajo de crestas secundarias.

Los dientes maxilares de Parksosaurus tienen varias crestas secundarias paralelas (Galton, 1973, siete en un diente figurado por Parks, 1926: fig. 1). Los dientes maxilares de Yandusaurus carecen de una cresta primaria y tienen entre siete y nueve crestas secundarias paralelas (He \& Cai, 1984). Los dientes maxilares de Zephyrosaurus tienen numerosas crestas secundarias (Sues, 1980; al menos hasta 10 según la figura 4 de Sues, 1980).

De Gongbusaurus y Qantassaurus no se conocen los dientes maxilares, sólo los mandibulares (Dong et al., 1983; Dong, 1989; Rich \& Rich, 1999), por lo que no es posible la comparación con los de El Peñascal.

En resumen, los dientes maxilares de El Peñascal se parecen a los de los "rhabdomorfos" Mochlodon, Muttabu- 
rrasaurus, Rhabdodon, Tenontosaurus, Zalmoxes, y a los de los "hipsilofodóntidos" Parksosaurus, Yandusaurus y Zephyrosaurus. Sin embargo, los dientes mandibulares de El Peñascal, con una cresta primaria muy desarrollada y con un máximo de 3 crestas secundarias poco marcadas, no se parecen a los dientes mandibulares de estos taxones (con numerosas crestas secundarias) ni a los de ninguno de los taxones de ornitópodos mencionados en la discusión.

Los dientes identificados como mandibulares son muy parecidos a los dientes mandibulares del ceratopsio Psitta-
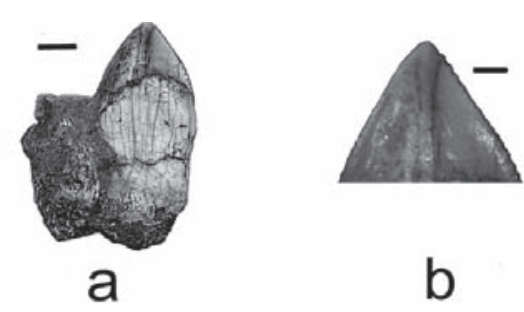

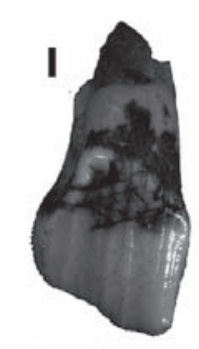

C

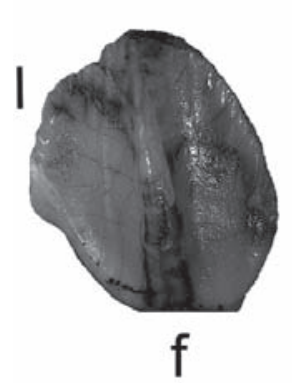

$f$

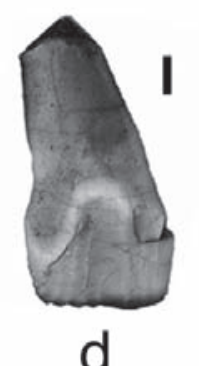

d
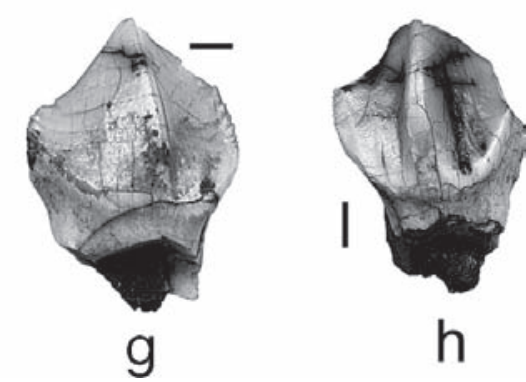

Figura 3. Dientes de Euornithopoda indet. de El Peñascal (Burgos). Vista labial en a, b, c, d, e; vista lingual en $\mathbf{f}, \mathbf{g}, \mathbf{h}$. a: diente premaxilar PS-PLS,21. b: detalle de la corona de PS-PLS,21. c: diente maxilar PS-PLS,18. d: diente maxilar PS-PLS, 25. e: diente maxilar PS-PLS, 34. f: corona del diente mandibular PS-PLS,19. g: diente mandibular PS-PLS,30. h: diente mandibular PS-PLS,35. Medida de referencia: $1 \mathrm{~mm}$.

Teeth of the Euornithopoda indet. from the El Peñascal site (Burgos, Spain). $\boldsymbol{a}, \boldsymbol{b}, \boldsymbol{c}, \boldsymbol{d}, \boldsymbol{e}$ : Labial views. $\boldsymbol{f}$, $\boldsymbol{g}$, $\boldsymbol{h}$ : Lingual views. $\boldsymbol{a}$ : Premaxilary tooth PS-PLS,21. b: Detail of the crown of PS-PLS,21. c: Maxillary tooth PS-PLS,18. d: Maxillary tooth PS-PLS,25. e: Maxillary tooth PS-PLS,34.f: Crown of the mandibular tooth PS-PLS,19. g: Mandibular tooth PS-PLS,30. $\boldsymbol{h}$ : Mandibular tooth PS-PLS,35. Scale bar: $1 \mathrm{~mm}$. cosaurus mongoliensis Osborn, 1923 (Osborn, 1923: figs. 4-5). Los psittacosáuridos (Psittacosaurus Osborn, 1923, y Hongshanosaurus You, Xu \& Wang, 2003) del Cretácico Inferior de Asia (China, Mongolia y Siberia) no tienen ninguna relación filogenética con los ornitópodos (Sereno, 2000), y además sus dientes maxilares son muy diferentes de los del euronitópodo de El Peñascal (Osborn, 1923; Sereno, 2000), por lo que la forma similar de los dientes mandibulares de éste es resultado de una convergencia.

\section{ANÁLISIS FILOGENÉTICO}

Weishampel et al. (2003) proponen 12 caracteres dentales en la sistemática de los ornitópodos no hadrosaurios (Weishampel et al., 2003, apéndice 3, caracteres 24-35), de los cuales 10 son aplicables al euornitópodo de El Penascal:

carácter 24: número de dientes premaxilares. Aunque no puede saberse cuántos, el euornitópodo de El Peñascal presentaba dientes premaxilares (estado primitivo), como Hypsilophodon y Tenontosaurus dossi (T. tilletti no presenta).

carácter 26: forma de los dientes maxilares. En el euornitópodo de El Peñascal la altura es $>50 \%$ de la longitud mesiodistal (estado derivado), como en Parksosaurus, Rhabdodon, Zalmoxes, Tenontosaurus, Dryosaurus, Camptosaurus Marsh, 1885 e Iguanodon.

carácter 27: crestas confluyentes con dentículos en los dientes maxilares. En el euornitópodo de El Peñascal hay abundantes crestas longitudinales que confluyen con los dentículos (estado derivado), como en Agilisaurus, Thescelosaurus, Parksosaurus, Hypsilophodon, Gasparinisaura, Rhabdodon, Zalmoxes, Tenontosaurus (contra Weishampel et al., 2003, véase Ostrom, 1970) y Dryosaurus.

carácter 28: cresta primaria en los dientes maxilares. En el euornitópodo de El Peñascal no está desarrollada (estado primitivo), como en Agilisaurus, Thescelosaurus, Parksosaurus, Hypsilophodon, Rhabdodon, Zalmoxes y Tenontosaurus.

carácter 29: esmalte en los dientes maxilares. En el euornitópodo de El Peñascal es asimétrico, más desarrollado en la cara labial (estado derivado), como en Hypsilophodon, Gasparinisaura, Rhabdodon, Zalmoxes, Tenontosaurus, Dryosaurus, Camptosaurus e Iguanodon.

carácter 31: forma de los dientes mandibulares. En el euornitópodo de El Peñascal la altura es $>50 \%$ de la longitud mesiodistal (estado derivado), como en Hypsilophodon, Rhabdodon, Zalmoxes, Tenontosaurus, Dryosaurus, Camptosaurus e Iguanodon.

carácter 32: número de crestas en los dientes mandibulares. En el euornitópodo de El Peñascal hay menos de 10 (estado primitivo), como en Agilisaurus, Thescelosaurus, Parksosaurus, Hypsilophodon, Gasparinisaura, Tenontosaurus, Dryosaurus, Camptosaurus e Iguanodon.

carácter 33: crestas confluyentes con dentículos en los dientes mandibulares. En el euornitópodo de El Peñascal no hay abundantes crestas longitudinales que confluyan con 
los dentículos (estado primitivo), como en Tenontosaurus, Dryosaurus, Camptosaurus e Iguanodon.

carácter 34: cresta primaria en los dientes mandibulares. En el euornitópodo de El Peñascal está desarrollada (estado derivado), como en Thescelosaurus, Hypsilophodon, Rhabdodon, Zalmoxes, Tenontosaurus, Dryosaurus e Iguanodon (contra Weishampel et al., 2003; véase Norman, 1980, 1986).

carácter 35: esmalte en los dientes mandibulares. En el euornitópodo de El Peñascal es asimétrico, más desarrollado en la cara lingual (estado derivado), como en Thescelosaurus, Hypsilophodon, Gasparinisaura, Rhabdodon, Zalmoxes, Tenontosaurus, Dryosaurus, Camptosaurus e Iguanodon.

El euornitópodo de El Peñascal presenta estado derivado en seis de estos caracteres $(26,27,29,31,34$ y 35$)$, y estado primitivo en el resto $(24,28,32$ y 33$)$. El euornitópodo de El Peñascal comparte estos seis caracteres derivados con Rhabdodon, Zalmoxes, Tenontosaurus y Dryosaurus. No obstante, las relaciones del euornitópodo de El Peñascal con estos cuatro taxones no quedan resueltas: Zalmoxes robustus, Tenontosaurus tilletti y Dryosaurus no presentan dientes premaxilares (carácter 24; en $Z$. shqiperorum se desconoce el valor de este carácter), diferenciándose del euornitópodo de El Peñascal; además Dryosaurus se diferencia por tener una cresta primaria en los dientes maxilares (carácter 28), y Rhabdodon y Zalmoxes por presentar más de 10 crestas longitudinales en los dientes mandibulares (carácter 32), que confluyan con los dentículos (carácter 33).

\section{COMPARACIÓN CON OTROS ORNITÓPODOS EUROPEOS}

En el Cretácico Inferior europeo se han definido varios ornitópodos: el heterodontosáurido Echinodon becklesii Owen, 1861 (Berriasiense de Inglaterra; Norman \& Barrett, 2002), el "hipsilofodóntido" Hypsilophodon foxii Huxley, 1869 (Barremiense de Inglaterra; Galton, 1974), los driosaúridos Valdosaurus canaliculatus (Galton, 1975) y "Camptosaurus" valdensis (Barremiense de Inglaterra; Galton, 1974, 1975; Galton \& Taquet, 1982), el camptosáurido Camptosaurus hoggii (Owen, 1874) (Berriasiense de Inglaterra; Norman \& Barrett, 2002), y varias especies de Iguanodon Mantell, 1825 (I. dawsoni Lydekker, 1889, e I. fittoni Lydekker, 1889 en el Valanginiense de Inglaterra; I. anglicus Holl, 1829 en el Valanginiense superior-Hauteriviense superior de Inglaterra, $I$. bernissartensis Boulenger, 1881 en el Hauteriviense-Aptiense de Bélgica, I. atherfieldensis Hooley, 1824 en el Barremiense-Aptiense de Inglaterra; Norman, 1980, 1986, 1987; Norman \& Weishampel, 1990).

Se desconoce la dentición de " $C$ ". valdensis, I. dawsoni e I. fittoni. El resto de taxones presentan una dentición diferente de la del euornitópodo de El Peñascal. Camptosaurus e Iguanodon carecen de dientes premaxilares (Norman \& Weishampel, 1990); además los dientes mandibulares de C. hoggii (los maxilares no se conocen) tienen dos crestas, primaria y secundaria, subiguales y paralelas, careciendo de crestas terciarias (Norman \& Barrett, 2002), y en Iguanodon spp. los dientes maxilares tienen una cresta primaria subcentral, muy prominente, y varias crestas secundarias, y los dientes mandibulares una cresta primaria subcentral, una cresta secundaria mesial y un número variable de crestas terciarias (Norman, 1980, 1986). La dentición de Valdosaurus Galton, 1977 no se conoce, excepto por un dentario asignado a este género (Galton \& Taquet, 1982), aunque por su gran parecido con Dryosaurus seguramente carecería de dientes premaxilares (Sues \& Norman, 1990); los dientes mandibulares de $V$. canaliculatus difieren de los de el euornitópodo de El Peñascal por presentar mayor número de crestas secundarias y carecer de cíngulo (Galton \& Taquet, 1982). E. becklesii tiene dientes maxilares y mandibulares con una cresta central y prominentes crestas mesial y distal, que conectan el dentículo más anterior y posterior con la base de la corona (Galton, 1978; Norman \& Barrett, 2002). Los dientes maxilares de $H$. foxii presentan menor número de crestas, hasta un máximo de seis, y los dientes mandibulares una cresta central menos marcada que los de El Peñascal (Galton, 1974).

Los dientes de El Peñascal difieren de los de Camptosaurus, Iguanodon, Echinodon Owen, 1861 e Hypsilophodon, y de los asignados a Valdosaurus, y representan un nuevo euornitópodo cuya posición sistemática es por el momento incierta.

\section{CONCLUSIONES}

Se describen dientes premaxilares, maxilares y mandibulares de ornitópodo del yacimiento del Cretácico Inferior (Barremiense superior-Aptiense) de El Peñascal (Salas de los Infantes, Burgos). Se ha comparado con todos los grupos de ornitópodos, encontrando que son de euornitópodo, y que podrían ser tanto de "hipsilofodóntido" como de "rhabdomorfo", aunque difieren de todos los géneros descritos de estos grupos, por lo que se determinan como Euornithopoda indet. Este taxón representa un nuevo euornitópodo para el Cretácico Inferior de Europa, posiblemente asignable a un nuevo género y especie.

\section{AGRADECIMIENTOS}

Al Área de Estratigrafía de la Universidad de Salamanca por fotografías realizadas con lupa binocular. La investigación de JIR-O forma parte del proyecto VECOBA (VErtebrados COntinentales del BArremiense, Ministerio de Ciencia y Tecnología, ref. BTE 2001-1746). Agradecemos a Xabier Pereda-Suberbiola (Universidad del País Vasco) y Francisco Ortega (Museo Jurá- 
sico de Asturias) las sugerencias que han contribuido a mejorar este trabajo.

\section{BIBLIOGRAFÍA}

Bakker, R. T., Galton, P., Siegwarth, J. \& Filla, J. 1990. A new latest Jurassic vertebrate fauna, from the highest level of the Morrison Formation at Como Bluff, Wyoming, with comments on Morrison biocronology. Hunteria, 2, 1-19.

Beuther, A., Dahm, H., Kneuper-Haack, F., Mensik, H., Tischer, G., Brinkmann, R., Dupuy de Lôme \& Herbert, L. 1966. Der Jura und Weladen in Nordost Spanien. Beihefte zum Geologischen Jahrbuch, 44, 224 pp..

Coria, R. A. \& Salgado, L. 1996. A basal iguanodontian (Ornithischia: Ornithopoda) from the Late Cretaceous of South America. Journal of Vertebrate Paleontology, 16, 445-457.

Dong Z. 1989. On a small ornithopod (Gongbusaurus wucaiwanensis) from Kelamaili, Junggar Basin, Xinjiang, China. Vertebrata PalAsiatica, 27, 140-146 (en chino con resumen en inglés, pp. 145-146).

Dong Z. \& Tang Z. 1983. Note on the new Mid-Jurassic ornithopod from Sichuan Basin, China. Vertebrata PalAsiatica, 21, 168-172 (en chino con resumen en inglés, pp. 172).

Dong Z., Zhou S. \& Zhang Y. 1983. The dinosaurian remains from Sichuan Basin, China. (Palaeontologia Sinica, Whole number 162, New Series C, 23). Science Press, Beijing, 1-145 (en chino con resumen en inglés, pp. 139-145).

Galton, P. M. 1973. Redescription of the skull and mandible of Parksosaurus from the Late Cretaceous with comments on the family Hypsilophodontidae (Ornithischia). Royal Ontario Museum Life Sciences Contribution, 89, 1-21.

Galton, P. M. 1974. The ornithischian dinosaur Hypsilophodon from the Wealden of the Isle of Wight. Bulletin of The British Museum (Natural History) Geology, 25, 1-152.

Galton, P. M. 1975. English hypsilophodontid dinosaurs (Reptilia: Ornitischia). Palaeontology, 48, 741-752.

Galton, P. M. 1978. Fabrosauridae, the basal family of ornithischian dinosaurs (Reptilia: Ornitischia). Paläontologische Zeitschrift, 52, 138-159.

Galton, P. M. 1983. The cranial anatomy of Dryosaurus, a Hypsilophodontid dinosaur from the Upper Jurassic of North America and East Africa, whit a review of hypsilophodontids from the Upper Jurassic of North America. Geologica et Palaeontologica, 17, 207-243.

Galton, P. M. 1995. The species of the basal hypsilophodontid dinosaur Thescelosaurus GILMORE (Ornithischia: Ornithopoda) from the late Cretaceous of North America. Neues Jahrbuch für Geologie und Paläontologie, Abhandlungen, 198, 297-311.

Galton, P. M. 1997. Cranial anatomy of the basal hypsilophodontid dinosaur Thescelosaurus GILMORE (Ornithischia: Ornithopoda) from the Upper Cretaceous of North America. Revue de Paléobiologie, 16, 231-258.

Galton, P. M. 1999. Cranial anatomy of the hypsilophodontid dinosaur Bugenasaura infernalis (Ornithischia: Ornithopoda) from the Upper Cretaceous of North America. Revue de Paléobiologie, 18, 517-534.

Galton, P. M. \& Taquet, P. 1982. Valdosaurus, a hypsilophodontid dinosaur from the Lower Cretaceous of Europe and Africa. Géobios, 15, 147-159.

He X. \& Cai K. 1984. The Middle Jurassic dinosaurian fauna from Dashampu, Zigong, Sichuan. Vol. 1: The ornitopod dinosaurs. Sichuan Scientific and Technological Publishing House, Chengdu, Sichuan, 1-71 (en chino con resumen en inglés; traducción al inglés disponible en The Polyglot Palaeontologist: http://ravenel.si.edu/paleo/paleoglot/).

Marsh, O. C. 1881. Principal characters of American Jurassic Dinosaurs. Part V. American Journal of Science (series 3), 21, 417-423.

Martín-Closas, C. \& Alonso Millán, A. 1998. Estratigrafía y bioestratigrafía (Charophyta) del Cretácico Inferior en el sector occidental de la Cuenca de Cameros (Cordillera Ibérica). Revista de la Sociedad Geológica de España, 11, 253-269.

Mas, J.R., Alonso, A. \& Guimerá, J., 1993. Evolución tectonosedimentaria de una cuencaextensional intraplaca: La cuenca finijurásica eocretácica de Los Cameros (La Rioja-Soria). Revista de la Sociedad Geológica de España, 6, 129-144.

Molnar, R. E. 1996. Observations of the australian ornithopod dinosaur, Muttaburrasaurus. In Proceedings of the Gondwanan Dinosaur Symposium (Eds. F. A. Novas \& R. E. Molnar). Memoirs of the Queensland Museum, 39, 639-652.

Norman, D. B. 1980. On the ornithischian dinosaur Iguanodon bernissartensis from the Lower Cretaceous of Bernissart (Belgium). Mémoires de l'Institute Royale des Sciences Naturelles de Belgique, 178, 1-105.

Norman, D. B. 1986. On the anatomy of Iguanodon atherfieldensis (Ornithischia: Ornithopoda). Bulletin de l'Institut Royal des Sciences Naturelles de Belgique Sciences de la Terre, 56, 281-372.

Norman, D. B. 1987. Wealden dinosaur biostratigraphy. In: Fouth Symposium on Mesozoic terrestrial ecosystems, Short Papers (Eds P. J. Currie \& E. H. Koster). Occasional Papers of the Tyrrel Museum of Palaentology, 3, 165-170.

Norman, D. B. 2001. A perspective of ornithopod dinosaurs: 1825-1999. In: Actas de las I Jornadas internacionales sobre Paleontología de Dinosaurios y su entorno (Ed. Colectivo Arqueológico-Paleontológico de Salas), Salas de los Infantes, 93-145.

Norman, D. B. 2004. Basal Iguanodontia. In: The Dinosauria, second edition (Eds. D. B. Weishampel, P. Dodson \& H. Osmólska). University of California Press, Berkeley, 413-437.

Norman, D. B. \& Barrett, P. M. 2002. Ornithischian dinosaurs from the lower Cretaceous (Berriasian) of England. Special Papers in Palaeontology, 68, 161-189.

Norman, D. B. \& Weishampel, D. B. 1990. Iguanodontidae and related Ornithopoda. In: The Dinosauria (Eds. D. B. Weishampel, P. Dodson \& H. Osmólska). University of California Press, Berkeley, 510-533.

Norman, D. B., Sues, H.-D., Witmer, L. M. \& Coria, R. A. 2004. Basal Ornithopoda. In: The Dinosauria, second edi- 
tion (Eds. D. B. Weishampel, P. Dodson \& H. Osmólska). University of California Press, Berkeley, 393-412.

Osborn, H. F. 1923. Two Lower Cretaceous dinosaurs from Mongolia. American Museum Novitates, 95, 1-10.

Ostrom, J. H.1970. Stratigraphy and Paleontology of the Cloverly Formation (Lower Cretaceous) of the Bighorn Basin Area, Wyoming and Montana. Bulletin of the Peabody Museum of Natural History, Yale University, 35, 1-234.

Parks, W. A. 1926. Thescelosaurus warreni, a new species of orthopodous dinosaur from the Edmonton formation of Alberta. University of Toronto Studies (Geological series), 21, 1-42.

Peng G. 1992. Jurassic ornithopod Agilisaurus louderbacki (Ornithopoda: Fabrosauridae) from Zigong, Sichian, China. Vertebrata PalAsiatica, 30, 39-53 (en chino con resumen en inglés, pp. 51-53).

Peng G. 1997. Fabrosauridae. In Encyclopedia of Dinosaurs (Eds. P. J. Currie \& K. Padian). Academic Press, San Diego, 237-240.

Pincemaille, M. 1997. Un ornithopode du Crétacé supérieur de Vitrolles (Bouches du Rhône), Rhabdodon priscus. Diplôme d'Etudes Approfondies, Université de Montpellier II, 1-46 (inédito).

Pincemaille, M. 1999. Discovery of a skeleton of Rhabdodon priscus (Ornithopoda, Dinosauria) in the Upper Cretaceous of Vitrolles (Bouches-du-Rhône, France). In: IV European Workshop on Vertebrate Paleontology, Albarracín (Teruel, Spain) Junio 1999. Programme and Abstracts, Field Guide (Eds. J. I. Canudo \& G. Cuenca-Bescós), 76.

Platt, N. H., 1986. Sedimentology and tectonics of the western Cameros Basin. Province of Burgos, Northern Spain. Thesis Oxford University, 1-125 (inédita).

Rich, T. H. V. \& Rich, P. V. 1989. Polar dinosaurs and biotas of Early Cretaceous of Southeastern Australia. National Geographic Research, 5, 15-53.

Rich, T. H. \& Vickers-Rich, P. 1999. The Hypsilophodontidae from southeastern Australia. In Proceedings of the Gondwanan Dinosaur Symposium (Eds. F. A. Novas \& R. E. Molnar). Memoirs of the Queensland Museum, 39, 167-180.

Ruiz-Omeñaca, J. I. 2001. Dinosaurios hipsilofodóntidos (Ornithischia: Ornithopoda) en la Península Ibérica. In: Actas de las I Jornadas internacionales sobre Paleontología de Dinosaurios y su entorno (Ed. Colectivo Arqueológico-Paleontológico de Salas), Salas de los Infantes, 175-266.

Seeley, H. G. 1887. On the classification of the fossil animals commonly named Dinosauria. Proceedings of the Royal Society of London, 43, 165-171.

Sereno, P. C. 1986. Phylogeny of the bird-hipped dinosaurs. National Geographic Research, 2, 234-256.

Sereno, P. C. 2000. The fossil record, systematics and evolution of pachycephalosaurs and ceratopsians from Asia. In: The Age of Dinosaurs in Russia and Mongolia (Eds. M. J. Benton, M. A. Shishkin, D. M. Unwin \& E. N.
Kurochkin). Cambridge University Press, Cambridge, 480-516.

Sues, H. D. 1980. Anatomy and relationships of a new hypsilophodontid dinosaur from the Lower Cretaceous of North America. Palaeontographica. Abteilung A: Palaeozoologie-Stratigraphie, 169, 51-72.

Sues, H. D. \& Norman, D. B. 1990. Hypsilophodontidae, Tenontosaurus, and Dryosauridae. In: The Dinosauria (Eds. D. B. Weishampel, P. Dodson \& H. Osmólska). University of California Press, Berkeley, 498- 509.

Torcida Fernández-Baldor, F., 1996. Registro de dinosaurios en el sureste de la provincia de Burgos. Zubía, 14, 89-104.

Torcida Fernández-Baldor, F., Ruiz-Omeñaca, J. I. Izquierdo Montero, L. A., Huerta Hurtado, P., Montero Huerta, D. \& Pérez Martínez, G., 2003. Nuevos restos de dinosaurios hipsilofodóntidos (Ornithischia: Ornithopoda) en el Cretácico Inferior de Burgos (España). En: Dinosaurios y otros Reptiles Mesozoicos en España (Coor. F. Pérez-Lorente). Instituto de Estudios Riojanos, Logroño, Ciencias de la Tierra, 26, 389-398.

Thulborn, R. A. 1970. The skull of Fabrosaurus australis, a triassic ornithischian dinosaur. Palaeontology, 13, 414432.

Thulborn, R. A. 1973. Teeth of ornithischian dinosaurs from the Upper Jurassic of Portugal, with description of a new hypsilophodontid (Phyllodon henkeli gen. et sp. nov.) from the Guimarota lignite. In: Contribuiçao para o conhecimento da fauna do Kimeridgiano da Mina de Lignito Guimarota (Leiria, Portugal). III Parte. Serviços Geológicos de Portugal, Memória no 22, (nova série), 89-134.

Weishampel, D. B.1984. Evolution of jaw mechanisms in ornithopod dinosaurs (Avdances in anatomy, embryology and cell biology, 87). Springer Verlag, Berlin, 1-110.

Weishampel, D. B. 1990. Ornithopoda. In: The Dinosauria (Eds. D. B. Weishampel, P. Dodson \& H. Osmólska). University of California Press, Berkeley, 484-485.

Weishampel, D. B., Jianu, C.-M., Csiki, Z. \& Norman, D. B. 2003. Osteology and phylogeny of Zalmoxes (n. g.), an unusual euornithopod dinosaur from the latest Cretaceous of Romania. Journal of Systematic Palaeontology, 1, 65-123.

Weishampel, D. B. \& Witmer, L. M. 1990. Heterodontosauridae. In: The Dinosauria (Eds. D. B. Weishampel, P. Dodson \& H. Osmólska). University of California Press, Berkeley, 486-497.

Zhao X., Cheng Z. \& Xu X. 1999. The earliest ceratopsian from the Tuchengzi Formation of Liaoning, China. Journal of Vertebrate Paleontology, 19, 681-691.

Manuscrito recibido: 19 de Febrero, 2004 Manuscrito aceptado: 19 de Mayo, 2005 